\title{
The Problem of a Hydroelasticity for a Tube Ring-type a Profile with Elastic, Geometrically Irregular Outer Shell at Pressure Influence
}

\author{
D. V. Kondratov ${ }^{1}$, Y. N. Kondratova ${ }^{2}$, V. S. Popov ${ }^{3}$, I. V. Plaksina ${ }^{4}$ \\ ${ }^{1}$ Stolypin Volga Region Institute Russian Academy of Public Administration under the President of the Russian Federation, Russia, \\ 410031, Saratov, Sobornaya st., 23/25, KondratovDV @ yandex.ru \\ ${ }^{2}$ Saratov State University, Russia, 410012, Saratov, Astrahanskaya st., 83, KondratovaUN@ info.sgu.ru \\ ${ }^{3}$ Saratov State Technical University, Russia, 410054, Saratov, Politekhnicheskaya st., 77, vic_p@bk.ru \\ ${ }^{4}$ Stolypin Volga Region Institute Russian Academy of Public Administration under the President of the Russian Federation, Russia, \\ 410031, Saratov, Sobornaya st., 23/25, chefirina@yandex.ru \\ The mechanical model presented in the form of a tube of ring section, formed by two surfaces of coaxial cylindrical shells cooperating \\ with viscous incompressible liquid is considered. The mathematical model of this system consisting of the differential equations \\ in private derivatives of describing dynamics of viscous incompressible liquid and an elastic ridge shell together with boundary \\ conditions is constructed.
}

Key words: hydroelasticity, viscous liquid, tube of ring section, geometrically irregular shell.

\section{References}

1. Bashta T. M. Mashinostroitel'naia gidravlika [Machine-building hydraulics]. Moscow, Mashgiz, 1963, 696 p. (in Russian).

2. Mogilevich L. I., Andrejchenko K. P. Dinamika giroskopov $s$ tsilindricheskim poplavkovym podvesom [Dynamics of gyroscopes with cylindrical floating suspension]. Saratov, Saratov Univ. Press, 1987, 160 p. (in Russian).

3. Mogilevich L. I., Popov V. S. Prikladnaia gidrouprugost' $v$ mashino- $i$ priborostroenii [Applied hydroelasticity in mechanical engineering and instrument making]. Saratov, 2003, 156 p. (in Russian).

4. Simdyankin A. A. Kontaktno-silovoe vzaimodeistvie detalei tsilindro-porshnevoi gruppy [The kontakt-force

interaction of details cylidr-piston group]. Saratov, 2003, 144 p. (in Russian).

5. Kondratov D. V., Mogilevich L. I. Mathematical modelling of processes of interaction of two cylindrical environments with the layer of the liquid between them under free leakage conditions of foundation vibration. Vestnik Saratovskogo gosudarstvennogo tekhnicheskogo universiteta, 2007, no. 3(26), iss. 1, pp. 22-31 (in Russian).

6. Mogilevich L. I., Popova A. A., Popov V. S. Dynamics's priests of interaction of an elastic cylindrical environment with a laminar stream of a liquid inside of it with reference to pipeline transport. Nauka i tekhnika transporta, 2007, no. 2, pp. 64-72 (in Russian).

УДК 501.1

\section{БИОМЕХАНИКА СОННОЙ АРТЕРИИ ЧЕЛОВЕКА С ПАТОЛОГИЧЕСКОЙ ИЗВИТОСТЬЮ}

\section{Л. Ю. Коссович ${ }^{1}$, К. М. Морозов ${ }^{2}$, О. Е. Павлова ${ }^{3}$}

\begin{abstract}
${ }^{1}$ Доктор фризико-математических наук, профрессор кафредры математической теории упругости и биомеханики, Саратовский государственный университет им. Н. Г. Чернышевского, nano-bio@sgu.ru

${ }^{2}$ Ведущий научный сотрудник отдела хирургии сосудов, Первый Московский государственный медицинский университет им. И. М. Сеченова, nano-bio@sgu.ru

${ }^{3}$ Инженер отдела биомеханики ОНИ наноструктур и биосистем, Саратовский государственный университет им. Н. Г. Чернышевского, Pavlovaoe@info.sgu.ru
\end{abstract}

Патологическая извитость сонной артерии занимает второе место в структуре причин сосудисто-мозговой недостаточности. Ранее авторами уже было описано влияние типа патологической извитости на поведение сонной артерии. В данной работе рассмотрено влияние различных анатомических (угол изгиба, размер ампулы) и реологических (уровень гематокрита) фракторов на гемодинамику и напряженно-десрормированное состояние сонной артерии с патологической извитостью. Выявлено, что уменьшение угла изгиба приводит к снижению объема крови, поступающей в мозг, и возможному фрормированию септального стеноза в области изгиба, а снижение уровня гематокрита способствует инициации процесса атерогенеза в зоне изгиба внутренней сонной артерии. Проведено численное моделирование реконструктивной операции на патологически извитой сонной артерии конкретного человека с атеросклеротическим поражением. Проведен расчет модели с предполагаемой геометрией сонной артерии данного пациента до возникновения патологий. Показано, что объемный кровоток после операции восстанавливается на 11\%, но не достигает значений для сонной артерии данного пациента в норме.

Ключевые слова: конечно-элементный анализ, патологическая извитость, сонная артерия, биомеханика. 


\section{ВВЕДЕНИЕ}

Сердечно-сосудистые заболевания являются одной из самых важных медицинских и социальных проблем в связи с их широкой распространенностью и тяжелыми последствиями для здоровья населения. Инсульт занимает второе место в структуре смертности, уступая лишь ишемической болезни сердца. В 2011 году в России было зарегистрировано около 882 тыс. случаев инсульта [1], из них около 333 тыс. привели к летальному исходу [2]. Необходимо отметить, что остаточные изменения встречаются у 30\% больных, перенесших инсульт, а трудоспособность восстанавливается лишь у $10 \%$ больных [3].

Основными причинами, приводящими к возникновению ишемического инсульта, являются атеросклероз и патологические извитости внутренних сонных и позвоночных артерий. Распространенность патологической извитости (ПИ) сонной артерии (СА) составляет около $26 \%$ среди людей, имеющих клинические симптомы или факторы риска сердечно-сосудистых заболеваний [4].

В соответствии с общепринятой классификацией выделяют четыре вида патологических извитостей: C- и S-образные изгибы, перегиб под острым углом и петле- и спиралеобразные извитости (рис. 1)

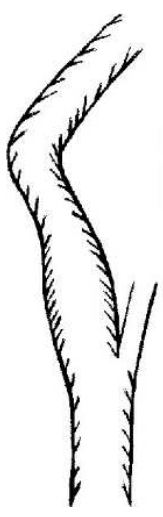

a



б

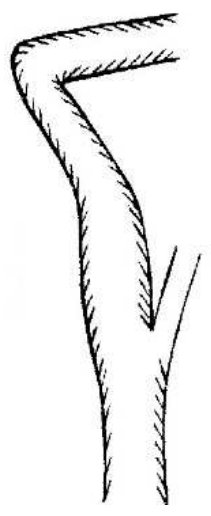

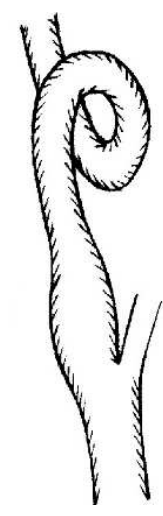

2

Рис. 1. Виды патологических извитостей: $a-$ С-образный изгиб, б - S-образный изгиб, в - перегиб, г - петля

Каждая форма извитости по-своему влияет на гемодинамику и напряженно-деформированное состояние сонной артерии. Результаты исследования влияния типа ПИ были описаны в предыдущих работах [5,6]. На поведение сонной артерии с конкретным видом ПИ оказывают влияние также размер ампулы СА, угол изгиба, уровень гематокрита в крови.

Таким образом, целью данной работы было биомеханическое исследование влияния перечисленных выше анатомических и реологических факторов на гемодинамические характеристики потока и напряженно-деформированное состояние стенок сосуда, а также последующее численное моделирование реконструктивной операции на сонной артерии с патологической извитостью для конкретного человека.

\section{1. МЕТОДЫ}

\section{1. Построение модели}

Построение трехмерных геометрических моделей сонных артерий с патологическими извитостями происходило на основе данных компьютерной томограммы с использованием специализированного программного пакета SolidWorks 2008 (SolidWorks corporation).

Исследование влияние анатомических и реологических факторов было проведено на примере сонной артерии с С-образным изгибом. Были построены модели СА в норме (рис. 2, a), с углом изгиба от $130^{\circ}$ до $90^{\circ}$ (рис. 2, б-е), с разными ампулами, отношение диаметра которой к диаметру сосуда составляло от 1 до 2.5 (рис. 2 , ж-к).

Для исследования влияния уровня гематокрита на гемодинамику сонной артерии с патологической извитостью использовалась геометрия, представленная на рис. 2 , б. 




$a$

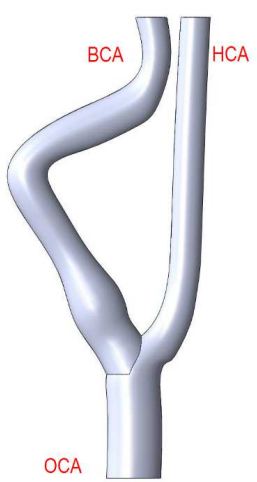

$e$

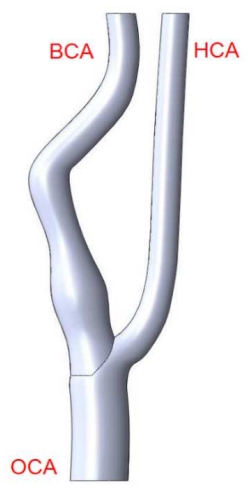

б

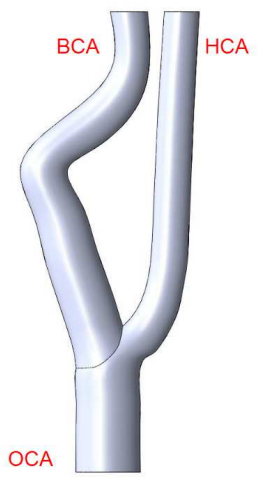

H
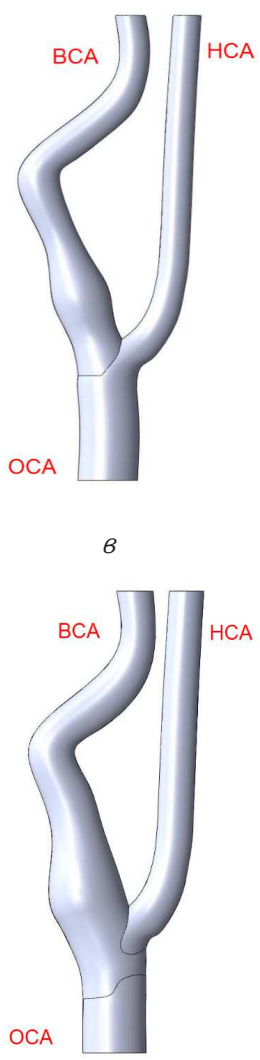

3
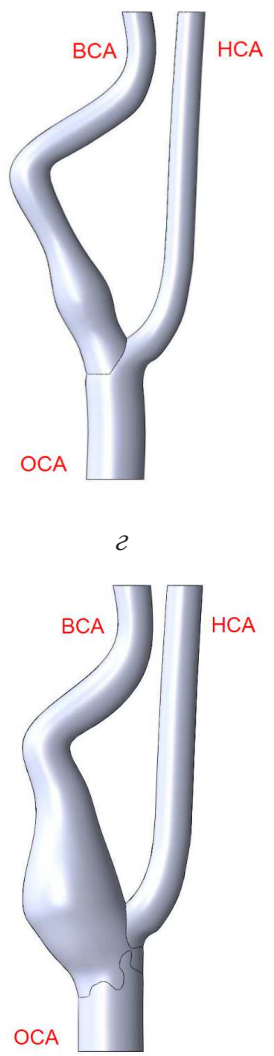

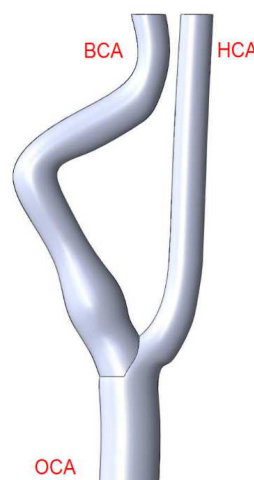

$\partial$

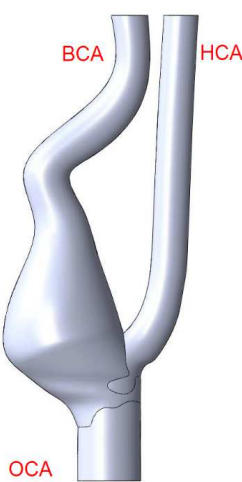


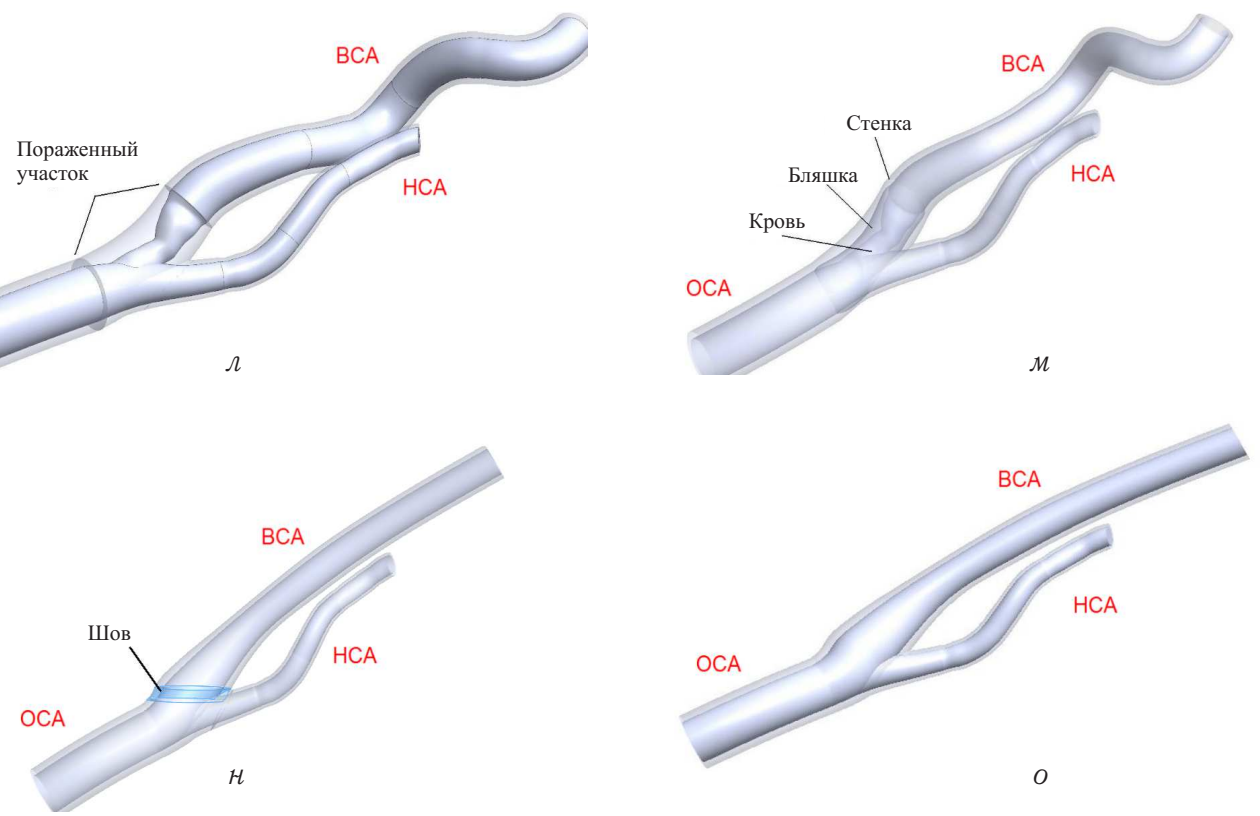

Рис. 2. Объемы, занимаемые кровью, для: $a-\mathrm{CA} \mathrm{в} \mathrm{норме,} \mathrm{б} \mathrm{-} \mathrm{СА} \mathrm{с} \mathrm{углом} \mathrm{изгиба} 130^{\circ}$, в - СА с углом изгиба $120^{\circ}, z-\mathrm{CA} \mathrm{с} \mathrm{углом} \mathrm{изгиба} 110^{\circ}, \partial-\mathrm{CA}$ суглом изгиба $100^{\circ}, e-\mathrm{CA} \mathrm{с} \mathrm{углом} \mathrm{изгиба} 90^{\circ}$, соотношением диаметра ампулы к диаметру сосуда 1 , з - СА с соотношением $1.5, u-$ СА с соотношением 2 , $\kappa-\mathrm{CA}$ с соотношением $2.5, \Omega-\mathrm{CA}$ с S-образной извитостью и пораженным атеросклерозом участком, $M-$ CA c S-образной извитостью и атеросклеротической бляшкой, $н-\mathrm{CA}$ после операции со швом, о - CA до 
Моделирование реконструктивной операции было выполнено для пациента, у которого сонная артерия была поражена атеросклерозом в области бифуркации общей сонной артерии (ОСА) и устья внутренней сонной артерии (BCA), сужающим просвет сосуда на 75\%, с последующей S-образной патологической извитостью ВСА. Было рассмотрено две модели. В первом случае пораженный атеросклерозом участок представлял собой один объем (модель 1 , рис. $2, \Omega$ ), во втором - два объема, соответствующих стенке и бляшке (модель 2, рис. 2, м). Была построена модель СА этого пациента после хирургического вмешательства (рис. 2, н) как описано в [7], но с выделением области шва в отдельное тело. Кроме того, была построена модель СА, предполагающая геометрию сосуда до возникновения патологий (рис. 2,0 ).

\section{2. Численный эксперимент}

Численные расчеты были проведены в конечно-элементном пакете Ansys (Ansys Inc., USA), который часто используется для решения задач биомеханики [8-10]. В качестве сетки для построенных геометрических моделей использовалось неструктурированное разбиение тетраэдрами ввиду сложности геометрии сосуда. Была исследована сеточная сходимость и выбран размер элемента 0.0003 м.

Кровь предполагалась ньютоновской жидкостью с плотностью $\rho=1050$ кг $/ \mathrm{m}^{3}$ и коэффициентом динамической вязкости $\mu=0.0037$ Па.с. Ее движение описывается уравнением Навье-Стокса для несжимаемой жидкости.

При исследовании влияния уровня гематокрита на гемодинамику и напряженно-деформированное состояние (НДС) сонной артерии с патологической извитостью значения вязкости и плотности крови изменялись, как показано в табл. 1.

На входе в сонную артерию задавалась функция скорости, на выходах из ВСА и наружной сонной артерии задавалось постоянное давление. При моделировании сонной артерии конкретного человека использовались значения скорости, полученные в результате допплерографического исследования данного пациента.

Материал стенки предполагался гиперупругим. Движение стенки описывается уравнением Навье-Ламе. Торцы сосуда были закреплены. Задавалось условие взаимодействия твердого тела и жидкости (Fluid-Solid Уровень гематокрита и соответствующие ему вязкость и плотность

\begin{tabular}{|c|c|c|}
\hline $\begin{array}{c}\text { Уровень гема- } \\
\text { токрита, \% }\end{array}$ & $\begin{array}{c}\text { Вязкость, } \\
\text { Па·с }\end{array}$ & $\begin{array}{c}\text { Плотность, } \\
\text { кг } / \mathrm{m}^{3}\end{array}$ \\
\hline 42 & 0.0039 & 1055 \\
\hline 35 & 0.0034 & 1051 \\
\hline 26 & 0.00275 & 1046 \\
\hline 20 & 0.0025 & 1040 \\
\hline
\end{tabular}
Interaction)

Для моделирования гиперупругого материала использовалась трехпараметрическая модель МуниРивлина. Кривые зависимости напряжения от деформации были взяты из литературных данных. При исследовании влияния анатомических и реологических факторов на гемодинамику и НДС сосуда были взяты результаты эксперимента на одноосное растяжение, приведенные в работе [11]. Для описания механических свойств стенки конкретного пациента и бляшки в модели 2 использовались данные из работы [12], пораженного атеросклерозом участка в модели 1 - результаты эксперимента на двухосное растяжение пораженной атеросклерозом ткани из работы [13]. Механические характеристики участка стенки, соответствующего шву, были взяты в виде $E=2 \cdot 10^{7} \mathrm{H} /$ м $^{2}$ (модуль Юнга) и $\rho=2500 \mathrm{kг} / \mathrm{M}^{3}$ (плотность).

\section{2. РЕЗУЛЬТАТЫ РАСЧЕТОВ}

\section{1. Исследование влияния анатомических и реологических фракторов}

Уменьшение угла изгиба приводит к уменьшению объема крови, поступающей в головной мозг. Для угла изгиба $90^{\circ}$ уменьшение среднего объемного кровотока за сердечный цикл по сравнению с нормой составляет 10\%. Увеличиваются максимальные значения касательных напряжений (KHC), эквивалентных напряжений (ЭН) и циклических деформаций (ЦД) в месте изгиба, локализующиеся на вогнутой стороне стенки изгиба (табл. 2), что вызывает повреждение стенки. таблица 2

Напряженно-деформированное состояние стенки в месте изгиба

\begin{tabular}{|c|c|c|c|}
\hline Модель & КНС, Па & ЭН, Па & ЦД \\
\hline норма & 18.53 & 22596 & 0.021 \\
\hline $130^{\circ}$ & 19.68 & 23014 & 0.0298 \\
\hline $120^{\circ}$ & 20.29 & 23591 & 0.0317 \\
\hline $110^{\circ}$ & 22.3 & 26069 & 0.0363 \\
\hline $100^{\circ}$ & 21.47 & 27901 & 0.0372 \\
\hline $90^{\circ}$ & 23.87 & 31156 & 0.0398 \\
\hline
\end{tabular}


Увеличение размера ампулы приводит к незначительному изменению объемного кровотока (менее 0.5\%). Средние значения давления, эквивалентных напряжений и циклических деформаций оказыва-

Таблица 3

Показатели гемодинамики и НДС в ампуле СА

\begin{tabular}{|l|c|c|c|c|}
\hline \multirow{2}{*}{ Параметры } & \multicolumn{4}{|c|}{ Соотношение диаметров ампулы и сосуда } \\
\cline { 2 - 5 } & 1 & 1.5 & 2 & 2.5 \\
\hline Давление, Па & 1410 & 1738 & 2171 & 2677 \\
\hline ЭН, Па & 23532 & 28962 & 35473 & 40464 \\
\hline ЦД & 0.0352 & 0.0414 & 0.0483 & 0.0507 \\
\hline
\end{tabular}
ются выше для большего размера ампулы (табл. 3), что может вызвать дальнейший рост ампулы.

Снижение уровня гематокрита вызывает незначительное уменьшение объемного кровотока на выходе из ВСА (менее $1.5 \%)$. Процент низких касательных напряжений (менее 1.5 Па) в месте изгиба на выпуклой стороне стенки увеличивается. Согласно гемодинамической теории атерогенеза области низких КНС являются благоприятным местом для развития атеросклероза.

\section{2. Моделирование реконструктивной операции}

Результаты численного анализа показали, что модель сонной артерии конкретного пациента, в которой пораженный атеросклерозом участок состоит из стенки и бляшки (модель 2 на рис. 2, м), лучше отражает имеющие место процессы. Систолическое значение скорости в месте сужения для модели № 1 составляет 1.84 м/с, а для модели $2-2.05$ м/с, что на $16.36 \%$ и 6.82\% соответственно меньше значения, полученного в ходе ультразвукового исследования. Для модели 2 значения давления выше и перепад давления в месте поражения СА атеросклерозом больше, чем для модели 1 на 187.1 Па (1.4 мм рт. ст.).

Низкие касательных напряжений стенки концентрируются сразу, после места максимального сужения сосуда и в местах изгиба на вогнутой стороне стенки. Максимальные значения КНС концентрируются на поверхности атеросклеротической бляшки, для модели 2 они на 2.21 Па выше, чем для модели 1.

Для модели 1 максимальные значения эквивалентных напряжений ниже на 5153 Па, чем для модели 2. Для модели 1 наблюдается повышение эквивалентных напряжений на стенке сосуда на стыке с пораженным атеросклерозом участком. Для модели 2 повышение значений ЭН наблюдается в зоне контакта сосудистой стенки и атеросклеротического поражения, что говорит о возможности ее отслоения.

В сонной артерии после хирургического вмешательства наблюдается нормальное распределение касательных напряжений на стенке сосуда, соответствующее распределению в сосуде до возникновения патологии. Максимальные значения эквивалентных напряжений концентрируются в апексе СА и более чем в два раза выше ЭН, наблюдающихся в сосуде до возникновения патологии. Высокие значения ЭН наблюдаются и в зоне шва, что может свидетельствовать о возможном рестенозе на данном участке сосуда.

Среднее значение объемного кровотока для модели 1 больше, чем для СА после реконструкции, что еще раз говорит в пользу модели 2. Средний объемный кровоток за сердечный цикл в СА после реконструкции увеличился на $11 \%$ по сравнению с моделью 2. Однако он остался меньше, чем для модели сосуда до образования патологий, разница составила около 15\%.

\section{выводы}

Исследовано влияние различных анатомических и реологических факторов на гемодинамику и напряженно-деформированное состояние сонной артерии с патологической извитостью. Показано, что уменьшение угла изгиба приводит к снижению объема крови, поступающей в мозг, и повреждению стенки сосуда в месте изгиба с возможным образованием септального стеноза. Увеличение размеров ампулы не меняет значительно объемного кровотока, но может вызвать дальнейший ее рост. Снижение уровня гематокрита повышает шансы возникновения атеросклеротического поражения в месте изгиба.

Для биомеханического моделирования реконструктивной операции на сонной артерии с патологической извитостью для конкретного пациента целесообразно использовать модель, в которой пораженный атеросклерозом участок представляет собой композицию из двух объемов, занимаемых стенкой и бляшкой с соответствующими им механическими свойствами. 
Выполненная операция позволяет восстановить объемный кровоток по внутренней сонной артерии на $11 \%$. Полученные данные согласуются с результатами ультразвуковых исследований сонной артерии для данного пациента.

\title{
Библиограсрический список
}

1. Заболеваемость взрослого населения России в 2011 году : стат. материалы : в 6 ч. / ФГБУ «ЦНИИОИЗ» Минздрава РФ. М., 2012. Ч. 3. 525 с.

2. Демографический ежегодник России. 2012. URL: http://www.gks.ru/bgd/regl/B12_16/Main.htm (дата обращения: 01.07.2013).

3. Спиридонов А. А., Лаврентьев А. В., Морозов К. М., Пирихалашщвили З. К. Микрохирургическая реваскуляризация каротидного бассейна. М. : Издво НЦССХ им. А. Н. Бакулева РАМН, 2000. 266 с.

4. Prencipe G., Pellegrino L., Vairo F., Tomaiuolo M., Furio O. A. Dolichoarteriopathy (kinking, coiling, tortuosity) of the carotid arteries and cardiovascular risk factors // Minerva Cardioangiol. 1998. Vol. 46, № 1-2. P. $1-7$.

5. Павлова О. Е., Иванов Д. В., Грамакова А. А., Морозов К. М., Суслов И. И. Гемодинамика и механическое поведение бифуркации сонной артерии с патологической извитостью // Изв. Сарат. ун-та. Нов. сер. Сер. Математика. Механика. Информатика. 2010. T. 10, № 2. C. 66-73.

6. Павлова О. Е., Иванов Д. В., Кириллова И. В. Численное исследование влияния патологической извитости артерии на кровоток // ANSYS Advantage. Русская версия. 2012. Вып. 18. С. 47-48.

7. Павлова O. E. Оперативное вмешательство на сонных артериях с патологией // Математическое моделирование и биомеханика в современном университете : тез. докл. VII Всерос. шк.-семинара. Ростов н/Д, 2012. C. 98 .

8. Голядкина А. А., Кириллова И. В., Щучкина О. А., Маслякова Г. Н., Островский Н. В., Челнокова Н. О. Конечно-элементное моделирование ишемической болезни сердца исходя из картины морфофункциональных изменений венечных артерий и сердечной мышцы человека // Рос. журн. биомеханики. 2011. Т. 15, № 4. С. 33-46.

9. Голядкина А. А., Кириллова И. В., Щучкина О. А. Анализ напряжённо-деформированного состояния и гемодинамики коронарных артерий и желудочков сердца человека // Вестн. Самар. гос. техн. ун-та. Сер. физ.-мат. науки. 2011. Т. 3, № 24. С. 79-88.

10. Голядкина А. А., Кириллова И. В. Численное моделирование напряженно-деформированного состояния стенок желудочков сердца человека в норме и при патологии // Вестн. Нижегород. ун-та им. Н. И. Лобачевского. 2011. Т. 4, № 2. С. 415-417.

11. Каменский A. В. Математическое моделирование поведения бифуркации сонной артерии человека на различных стадиях атеросклеротического поражения и после операционного вмешательства: дис. ... канд. физ.мат. наук. Саратов, 2007. 179 с.

12. Wu Wei, Qi Min, Liu Xiao-Peng Yang, Da-Zhi, Wang Wei-Qiang. Delivery and release of nitinol stent in carotid artery and their interactions: A finite element analysis // J. Biomech. 2007. Vol. 40. P. 3034-3040.

13. Kural M. H., Cai M., Tang D., Gwyther T., Zheng J., Billiar K. L. Planar biaxial characterization of diseased human coronary and carotid arteries for computational modeling // J. Biomech. 2012. Vol. 45. P. 790-798.

\section{Biomechanics of Human Carotid Artery with Pathological Tortuosity}

\section{Yu. Kossovich ${ }^{1}$, K. M. Morozov² ${ }^{2}$ O. E. Pavlova ${ }^{3}$}

\author{
${ }^{1}$ Saratov State University, Russia, 410012, Saratov, Astrahanskaya st., 83, nano-bio@sgu.ru \\ ${ }^{2}$ I. M. Sechenov First Moscow State Medical University, Russia, 119991, Moscow, Trubetskaya st., 8-2, nano-bio@sgu.ru \\ ${ }^{3}$ Saratov State University, Russia, 410012, Saratov, Astrahanskaya st., 83, Pavlovaoe@ info.sgu.ru
}

Pathological tortuosity of carotid artery ranks second among the causes of cerebro-vascular insufficiency. In their previous researches authors have described the influence of pathological tortuosity type on carotid artery behaviour. In this article we discuss the influence of different anatomic (bending angle, bulb size) and rheological factors on haemodynamics and stress-strain state of carotid artery with pathological tortuosity. Decreasing of the bending angle leads to blood volume reduction in brain and possible formation of septal stenosis in bending area. Diminution of hematocrit level promotes the initiation of atherogenesis in internal carotid bend. Computational modeling of reconstructive surgery of pathologically tortuous carotid of the patient with atherosclerosis was carried out. Numerical analysis of plausible model of patient carotid before pathology formation was conducted. Volume blood flow after surgery has increased by $11 \%$, but remained to be lower than in the healthy patient.

Key words: finite-element analysis, pathological tortuosity, carotid artery, biomechanics. 


\section{References}

1. Zabolevaemost' vzroslogo naseleniia Rossii v 2011 godu [Morbidity rate of adult population of Russia in 2011]. Moscow, 2012, pt. 3, 525 p. (in Russian).

2. Demographical Annual of Russia. 2012. Available at: http://www.gks.ru/bgd/regl/B12_16/Main.htm (Accessed 01, July, 2013).

3. Spiridonov A. A., Lavrent'ev A. V., Morozov K. M., Pirtskhalaishvili Z. K. Mikrokhirurgichsekaia revaskuliarizatsiia karotidnogo basseina [Microsurgical revasculization of carotid system]. Moscow, 2000, 266 p. (in Russian).

4. Prencipe G., Pellegrino L., Vairo F., Tomaiuolo M., Furio O. A. Dolichoarteriopathy (kinking, coiling, tortuosity) of the carotid arteries and cardiovascular risk factors. Minerva Cardioangiol, 1998, vol. 46, no. 1-2, pp. 1-7.

5. Pavlova O. E., Ivanov D. V., Gramakova A. A., Morozov K. M., Suslov I. I. Hemodynamics and Mechanical Behavior of Pathologically Tortuous Carotid Arteries. Izv. Sarat. Univ. N.S. Ser. Math. Mech. Inform., 2010, vol. 10, iss. 2, pp. 66-73 (in Russian).

6. Pavlova O. E., Ivanov D. V., Kirillova I. V. Computational Investigation of Pathological Tortuosity Influence on artery bloodflow. Ansys Advantage. Russian Edition., 2012, iss. 18, pp. 47-48 (in Russian).

7. Pavlova O. E. Operativnoe vmeshatel'stvo na sonnykh arteriiakh s patologiei [Surgical Treatment of Carotid Arteries with Tortuosity]. Matematicheskoe modelirovanie $i$ biomekhanika v sovremennom universitete : tezisy dokladov VII Vserossiiskoi shkoly-seminara, Rostov-onDon, 2012, pp. 98 (in Russian).

8. Golyadkina A. A., Kirillova I. V., Schuchkina O. A.,
Maslyakova G. N., Ostrovskiy N. V., Chelnokova N. O. The Finite Element Modelling of Ischemic Heart Disease Based on the Morphological and Functional Changes of Human Coronary Arteries and Myocardium. Russian Journal of Biomechanics, 2011, vol. 15, iss. 4, pp. 3346 (in Russian).

9. Golyadkina A. A., Kirillova I. V., Schuchkina O. A. Investigation of Human Coronary Arteries and Heart Ventricles Mode of Deformation and Haemodynamics Vestnik SamGTU. Ser. Fiz.-Math. Nauk., 2011, vol. 3, iss. 24 , pp. $79-88$ (in Russian).

10. Golyadkina A. A., Kirillova I. V. Numerical Modeling of Stress-streain State of Normal and Pathological Human Ventricle Walls Vestnik of Lobachevsky State University of Nizhni Novgorod, 2011, vol. 4, iss. 2, pp. 415-417 (in Russian).

11. Kamenskiy A. V. Matematicheskoe modelirovanie povedeniia bifurkatsii sonnoi arterii cheloveka na razlichnykh stadiiakh ateroskleroticheskogo porazheniia $i$ posle operatsionnogo umeshatel'stva. Diss. kand. fiz.mat. nauk [Mathematical modeling of carotid bifurcation behaviour at different stages of atherosclerosis and after surgical treatment: Cand. phys. and math. sci. diss.]. Saratov, 2007. 170 p. (in Russian).

12. Wu Wei, Qi Min, Liu Xiao-Peng Yang, Da-Zhi, Wang Wei-Qiang. Delivery and release of nitinol stent in carotid artery and their interactions: A finite element analysis. $J$. Biomech., 2007, vol. 40, pp. 3034-3040.

13. Kural M. H., Cai M., Tang D., Gwyther T., Zheng J., Billiar K. L. Planar biaxial characterization of diseased human coronary and carotid arteries for computational modeling. J. Biomech., 2012, vol. 45, pp. 790-798. 